\title{
NONRECURSIVE FUNCTIONS IN REAL ALGEBRAIC GEOMETRY
}

\author{
ALEXANDER NABUTOVSKY
}

\begin{abstract}
As a result of the Tarski-Seidenberg theorem, problems in real algebraic geometry usually have constructive solutions. In this article we show that this is not always the case. We consider the following problem, which is of interest for its own sake.

Let $\Sigma^{n} \subset \mathbf{R}^{n+1}$ be a nonsingular compact algebraic surface of degree $d$. Let $\Sigma^{n}$ be isotopic to the standard hypersphere $S^{n} \subset \mathbf{R}^{n+1}$. It is well known that it is not always possible to connect $\Sigma^{n}$ and $S^{n}$ by an isotopy passing via nonsingular algebraic hypersurfaces of degree not higher than $d$. We prove that it is always possible to connect $\Sigma^{n}$ and $S^{n}$ by an isotopy passing via algebraic surfaces of some degree $A$ which depends on $n$ and $d$ only. Consider for each $n$ the smallest of such degrees as a function $A_{n}(d)$. What can be said about these functions?

We prove that for each $n \geq 5$, the function $A_{n}(d)$ cannot be majorized by a recursive function of $d$. Also, some generalizations of these results are stated below.
\end{abstract}

1. Algebraic approximation of isotopies. It is well known that a compact hypersurface in a Euclidean space may be approximated by a nonsingular algebraic hypersurface. It is possible to prove that an isotopy between two compact nonsingular algebraic hypersurfaces may be approximated by an isotopy which passes via nonsingular algebraic hypersurfaces only. This can be generalized for regular complete intersections of compact nonsingular algebraic hypersurfaces. More precisely, let $p_{i}(x)$, $i \in\{1, \ldots, k\}$ be a system of polynomials on $\mathbf{R}^{n+k}$ such that for all $i$ the set $Z_{i}$ of zeroes of $p_{i}(x)$ is compact and not empty and the Jacobi matrix $J\left(p_{1}(x), p_{2}(x), \ldots, p_{k}(x)\right)$ has maximal rank at each point of $Z_{p}=Z_{1} \cap Z_{2} \cap \cdots \cap Z_{k}$. If $Z_{p}$ is nonempty, then we call $\left\{p_{i}(x)\right\} a$ regular system and $Z_{p}$ a compact complete intersection, or briefly a CCI (cf. [1, 4] and references there for properties of compact complete intersections). If some CCI $M^{n} \subset \mathbf{R}^{n+k}$ is representable as a regular intersection of nonsingular compact algebraic hypersurfaces of degrees less than or equal to $d$, then we shall call $M^{n}$ a d-CCI. Let $\Omega_{n, k}\left(M^{n}\right)$ denote the space of all $n$-dimensional submanifolds of $\mathbf{R}^{n+k}$, diffeomorphic to $M^{n}$, with the $C^{1}$-topology. We shall denote by $T_{n}\left(\mathbf{R}^{n+k}\right)$ the space $\bigvee \Omega_{n, k}\left(M^{n}\right)$, where the disjoint union is taken over all nondiffeomorphic compact $C^{\infty}$-smooth manifolds $M^{n}$ embeddable in $\mathbf{R}^{n+k}$, and by $T_{n}^{(d)}\left(\mathbf{R}^{n+k}\right)$ its subspace consisting of all d-CCI's. We shall say that two

Received by the editors January 27, 1988.

1980 Mathematics Subject Classification (1985 Revision). Primary 14F45, 14N99, 03D35; Secondary 14M10, 14J99, 57R52, 41A10. 
d-CCI's, $M_{1}^{n}$ and $M_{2}^{n}$ are $D$-rigidly isotopic if there exists some continuous mapping $F^{*}:[0,1] \rightarrow T_{n}^{(D)}\left(\mathbf{R}^{n+k}\right)$ (which will be called a $D$-rigid isotopy), such that $F^{*}(0)=M_{1}^{n}, F^{*}(1)=M_{2}^{n}$. For $k=1$, this is a generalization and a modification of the rigid isotopy definition by V. A. Rokhlin [9]; unlike him, we permit singularities in the complex nonreal part of the zero set of polynomials during the isotopy and also permit the degrees of the polynomials to increase from $d$ to $D$ during the isotopy. Obviously "to be $D$-rigidly isotopic" is an equivalence relation on $T_{n}^{(d)}\left(\mathbf{R}^{n+k}\right)$.

In such terms, for $k=1$, Proposition 1.1 says that if two compact non-singular algebraic hypersurfaces are. isotopic then they are $D$-rigidly isotopic for some $D$.

Proposition 1.1. Let $F:[0,1] \rightarrow T_{n}\left(\mathbf{R}^{n+k}\right)$ be a smooth isotopy such that $F(0), F(1) \in T_{n}^{(d)}\left(\mathbf{R}^{n+k}\right)$. Then there exist some $D$ and a smooth isotopy $F^{*}:[0,1] \rightarrow T_{n}^{(D)}\left(\mathbf{R}^{n+k}\right)$ such that $F^{*}(0)=F(0), F^{*}(1)=F(1)$.

Proof (for $k=1$ ). For each $t$, approximate $F(t)$ constructively by a nonsingular algebraic hypersurface $A_{0}(t)=\left\{x \in \mathbf{R}^{n+1} \mid p_{0 t}(x)=0\right\}$ (cf. [6, 10]) in such a manner that $p_{0 t}(x)$ be positive for large enough $\|x\|$ and $A_{0}(i)=F(i), i \in\{0,1\}$. It is possible to prove that for a close enough approximation there exists some finite set of $t\left\{t_{0}, t_{1}, \ldots, t_{m-1}, t_{m}\right\}, t_{0}=0$, $t_{m}=1$, such that for each $t \in\left[t_{i}, t_{i+1}\right]$ the set

$$
\begin{array}{r}
A(t)=\left\{x \in \mathbf{R}^{n+1} \mid\left(\left(t-t_{i}\right) /\left(t_{i+1}-t_{i}\right)\right) p_{0 t_{i+1}}(x)\right. \\
\left.+\left(\left(t_{i+1}-t\right) /\left(t_{i+1}-t_{i}\right)\right) p_{0 t_{i}}(x)=0\right\}
\end{array}
$$

is a nonsingular algebraic hypersurface. The necessary isotopy $F^{*}$ is obtained by the canonical projection of the resulting broken line in the space of vectors of polynomial coefficients on $T_{n}^{(d)}\left(\mathbf{R}^{n+1}\right)$. Q.E.D.

The following theorem states that the number $D$ in the statement of Proposition 1.1 can be chosen to be independent of the subvarieties $F(0)$, $F(1)$ and dependent only on $n$ and a given upper bound $d$ of degrees $F(0)$ and $F(1)$. Thus it is a strengthened form of Proposition 1.1.

Theorem 1.2. For each positive integer $d, n, k$ there exists $D$ such that each two isotopic n-dimensional d-CCI's in $\mathbf{R}^{n+k}$ are D-rigidly isotopic.

Proof. Consider the set $\Phi_{n, k, d}$ of all regular systems of $k$ polynomials of $n+k$ variables of degree less than or equal to $d$. Fix some numeration of all $\left(\begin{array}{c}n+k+d \\ d\end{array}\right) k$ monomial coefficients in such systems. Now we can consider $\Phi_{n, k, d}$ as a subset of $\left.\mathbf{R}^{(n+k+d}\right)^{k}$. The set $\Phi_{n, k, d}$ is a semialgebraic subset of the Euclidean space. Hence it has a finite number of connected components (cf. [3]). The CCI's represented by a given connected component of $\Phi_{n, k, d}$ are isotopic. Choose one representative in each connected component of $\Phi_{n, k, d}$. Denote the corresponding CCI's by $C_{i}$. Each d-CCI is d-rigidly isotopic to one of the constructed CCl's $C_{i}$. Let $C_{i_{1}}$ be isotopic to $C_{i_{2}}$ for some $i_{1}, i_{2}$. Proposition 1.1 implies that there exists a number $d_{i_{1} i_{2}}$ such that $C_{i_{1}}$ and $C_{i_{2}}$ are $d_{i_{1} i_{2}}$-rigidly isotopic. Let $I=\left\{(i, j) \mid C_{i}\right.$ and $C_{j}$ 
are isotopic $\}, D_{0}=\max _{(i, j) \in I} d_{i j}, D=\max \left\{d, D_{0}\right\}$. Obviously $D$ is the necessary number. Q.E.D.

Denote the minimal value of such $D$ for some fixed $d, n, k$ by $A_{n, k}(d)$. We shall consider below these numbers as functions of $d$ (for each fixed pair of $n, k$ ).

Each pair of $D$-rigidly isotopic $n$-dimensional CCI's in $\mathbf{R}^{n+k}$ can be connected by a $D$-rigid isotopy which can be represented by a piecewisecontinuous path in the set $\Phi_{n, k, D}$ of vectors of coefficients of regular systems. Moreover, this isotopy can be chosen in such a manner that each connected component of $\Phi_{n, k, D}$ will contain no more than one piece of the path. Hence, the semialgebraicity of $\Phi_{n, k, D}$ makes it possible to majorize effectively the number of the pieces (for example, using results of [8]). This makes it possible to prove the following result, using the Tarski-Seidenberg theorem.

THEOREM 1.3. There exists an algorithm which verifies for each set of given $n, k, d, D$ and for each given pair of vectors with rational components representing some d-CCI's $M_{1}^{n}, M_{2}^{n} \subset \mathbf{R}^{n+k}$, whether $M_{1}^{n}$ and $M_{2}^{n}$ are $D$ rigidly isotopic in $\mathbf{R}^{n+k}$.

2. Nonrecursive functions in real algebraic geometry. Let $M^{n} \subset \mathbf{R}^{n+1}$ be a $C^{\infty}$-smooth manifold, and $n \geq 5$. We are interested in the question: Is $M^{n}$ diffeomorphic to the standard sphere $S^{n}$ or not? Provided that $M^{n}$ is represented in a standard way described in [2] or in an equivalent one, there is no algorithm which provides an answer to this question (this is a result of S. P. Novikov, cf. its exposition in [11, $\S 10]$ ). More precisely, S. P. Novikov constructed a sequence $\Sigma_{i}^{n} \subset \mathbf{R}^{n+1}, i \in \mathbf{N}$, of smooth hypersurfaces such that the problem of $n$-dimensional sphere recognition is undecidable even for this sequence. We can constructively approximate $M^{n}$ by an isotopic nonsingular algebraic hypersurface $A^{n} \subset \mathbf{R}^{n+1}$, $A^{n}=\left\{x \in \mathbf{R}^{n+1} \mid p(x)=0\right\}, \operatorname{grad} p(x) \neq 0$ on $A^{n}$, all coefficients of $p(x)$ are rational. "Constructively" means that all the coefficients of one such polynomial $p$ can be found from a data set describing $M^{n}$. Hence, the recognition problem, whether or not $M^{n}$ is diffeomorphic to a standard sphere $S^{n}$, is undecidable even in the class of nonsingular algebraic manifolds $M^{n}$ which can be represented as nonsingular zero sets of polynomials with rational coefficients $p: \mathbf{R}^{n+1} \rightarrow \mathbf{R}$, providing $M^{n}$ is given by a vector of coefficients of one such polynomial. Further, it is well known that there are no nontrivial smooth codimension one knots in $\mathbf{R}^{n+1}(n \geq 4)$ (cf. [7, Chapter 9, Proposition D]). Hence a smooth hypersurface in $\mathbf{R}^{n+1}(n \geq 4)$ is isotopic to a standard sphere $S^{n} \subset \mathbf{R}^{n+1}$ if and only if the hypersurface is diffeomorphic to $S^{n}$. This implies that "diffeomorphic" in the formulation of the problem above for hypersurfaces is equivalent to "isotopic." Thus, we obtain the codimension one case $(k=1)$ of the following proposition.

Proposition 2.1. Let $n \geq 5$ and arbitrary $k$ be fixed. There is no algorithm which permits to determine for a given ordered set of rational coefficients of some polynomial mapping $p: \mathbf{R}^{n+k} \rightarrow \mathbf{R}^{k}$, such that its zero set $Z_{p}$ 
is not empty and is nonsingular, whether or not $Z_{p}$ is isotopic to the sphere $S^{n}=\left\{x \in \mathbf{R}^{n+k} \mid x_{n+2}=\cdots=x_{n+k}=0,\|x\|=1\right\}$.

The general case of the proposition can be easily deduced from the codimension one case.

We can now state our main result. But first recall that $A_{n, k}(d)$ denotes the minimal number $D \in \mathbf{N}$ such that any two isotopic d-CCI's of dimension $n$ and codimension $k$ are $D$-rigidly isotopic. Theorem 1.2 ensures the existence of $A_{n, k}(d)$. Define $A_{n, k}^{S^{n}}(d)$ as the minimal number $E \in \mathbf{N}$ such that any two d-CCI's of dimension $n$ and codimension $k$ which are isotopic to the standard sphere $S^{n}$ are $E$-rigidly isotopic. Obviously $A_{n, k}^{S^{n}}(d) \leq A_{n, k}(d)$.

MAIN TheOREM. For each $n \geq 5$ and for each $k$ the function $A_{n, k}^{S^{n}}(d)$ cannot be majorized by any recursive function of $d$.

Proof. We prove the theorem by contradiction. Assume that for some $n \geq 5, k$, the function $A_{n, k}^{S^{n}}(d)$ can be majorized by some recursive function $\varphi(d)$. By Theorem 1.3 and by recursiveness of $\varphi(d)$ there exists an algorithm for the decision problem whether or not two d-CCl's $M_{1}^{n}, M_{2}^{n}$ are $\varphi(d)$-rigidly isotopic. But, by the definition of $A_{n, k}^{S^{n}}(d)$, for each $n$ dimensional d-CCI $M^{n} \subset \mathbf{R}^{n+k}$, the existence of an isotopy connecting $M^{n}$ and the 2-CCI $S^{n}$ is equivalent to the existence of a connecting $\varphi(d)$-rigid isotopy. Thus our assumption contradicts Proposition 2.1. This proves the theorem. Q.E.D.

What can be said about the functions $A_{n, k}(d)\left(A_{n, k}^{S^{n}}(d)\right)$ for $n \leq 4$ ?

We have proved using the same method, that for each $k \geq 4$ the function $A_{4, k}(d)$ cannot be majorized by any recursive function of $d$. In [2] a sequence of hypersurfaces in $\mathbf{R}^{5}$ was constructed in order to prove the algorithmic unsolvability of the diffeomorphism problem. We used this sequence instead of the S. P. Novikov's sequence to prove an analogue of Proposition 2.1 which is valid for $n=4$. The constraint $k \geq 4$ arises because of the necessity to use also the following particular case of Haefliger's theorem [5, p. 47]: Any two diffeomorphic 4-dimensional connected, simply connected submanifolds of $\mathbf{R}^{4+k}$ are isotopic if $k \geq 4$.

However, one can prove easily that $A_{1,1}(d)$ is a recursive function.

Acknowledgements. I would like to thank Professors A. I. Fet, O. Viro, N. Ivanov, V. Kharlamov, A. Slisenko, B. Moishezon, Y. Yomdin, S. Kiro, V. Schuchman, M. Farber and Dr. G. Weiss for very helpful discussions. I would like to thank Professor J. Bochnak for sending me a copy of the preprint [1].

\section{REFERENCES}

1. J. Bochnak and W. Kucharz, On complete intersections in differential topology and analytic geometry, preprint, 1983.

2. W. W. Boone, W. Haken and V. Poénaru, On recursively unsolvable problems in topology and their classification, Contributions to Mathematical Logic (H. Arnold Schmidt, K. Schutte, H.-J. Thiele, eds.) North-Holland, Amsterdam, 1968. 
3. M. Coste, Ensembles semi-algébriques, Géométrie Algébrique Réelle et Formes Quadratiques, Journées S.M.F. Université de Rennes 1, Mai 1981, (J.-L. Colliot-Théléne, M. Coste, L. Mahé, M.-F. Roy, eds.) Lecture Notes in Math., vol. 959, Springer-Verlag, Berlin and New York, 1982, pp. 109-138.

4. O. Forster, Complete intersections in affine algebraic varieties and Stein spaces, Complete Intersections, (S. Greco and R. Strano, eds.) Lecture Notes in Math., vol. 1092, SpringerVerlag, Berlin and New York, 1984, pp. 1-28.

5. A. Haefliger, Plongement différentiables de variétés dans variétés, Comment. Math. Helv. 36 (1962), 47-82.

6. N. V. Ivanov, Approximation of smooth manifolds by real algebraic sets, Russian Math. Surveys 37 (1982), 3-52.

7. J. Milnor, Lectures on the h-cobordism theorem, Princeton Univ. Press, Princeton, N.J., 1965.

8.

9. V. A. Rokhlin, Complex topological characteristics of real algebraic curves, Russian Math. Surveys 33 (1978), 85-98.

10. H. Seifert, Algebraische approximation von Manningfaltigkeiten, Math. Z. 41 (1936), $1-17$.

11. I. A. Volodin, V. E. Kuznetsov and A. T. Fomenko, The problem of discriminating algorithmically the standard three-dimensional sphere, Russian Math. Surveys 29 (1974), 71172.

Department of Theoretical Mathematics, The Weizmann Institute of Science, P.O.B. 26, REHOVOT 76100, ISRAEL 
